\title{
Removal of Drugs from Hospital Wastewater by Photodegradation
}

\author{
Type of article: Conference abstract \\ Assia Keniche1,2, Leshaf Anissa1, Mazari Oussama1, \\ 1Maghnia Center University, Tlemcen, Algeria \\ 2Laboratory of Organic Chemistry, Natural Products, and Analysis, University of Tlemcen, \\ Tlemcen, Algeria \\ Corresponding Author: keniche_assia@yahoo.fr
}

\begin{abstract}
:
Background: Hospital wastewater (WWs) represents a particular type of effluent, compared with urban wastewater. Hospitals generate on average $750 \mathrm{~L}$ of wastewater by the bed and by day so they are 2-5 times higher than urban flow rates, which refer to one inhabitant equivalent (typically included in the interval 120-250 L). This significant quantity of water per day for different purposes and services depending on the activities which take place within the structure.

Methods: In our work, we proposed two methods; the first one is the complexation with CD for the treatment of drugs from Hospital WWs, which makes solid precipitate that can be filtered later. Our second method is the photodegradation of most drugs found in Hospital WWs with a UV lamp and time for each type of drug elimination.

Results: Our result for the first method is the formation of a CD-drug complex as a solid precipitate which will be filtered later and eliminate the solution to be purified. For the second method, the use of the UV lamp at $300 \mathrm{~nm}$ gave a degradation of $70 \%$ of the drug of the solution after 30 minutes of irradiation. The follow-up of the photodegradation was carried out by UV spectroscopy.

Conclusion: The elimination of drugs from hospital waters is essential, the method we have developed on a small scale can be standardized in Algerian hospitals that do not contain hospital water treatment policies, before reaching urban waters. This congress will be the occasion to discuss this national problem and a call of installation of ministry at the level of each hospital.
\end{abstract}

Keywords: Hospital Water, Wastewater, Photodegradation, UV Spectroscopy, Water Treatment.

\section{Conflict of interest statement}

This article is a conference abstract presented at the International Congress on Health Sciences and Medical Technologies, Tlemcen Algeria 5-7 December 2019, ICHSMT' 19.

\section{Authors' biography}

No Biography. 
Medical Technologies Journal, Volume: 4, Issue: 1, January-March 2020, Pages: 523-524. Doi :

https://doi.org/10.26415/2572-004X-vol4iss1p523-524

\section{References}

No references 